\title{
Active management of the third stage of labour with and without controlled cord traction: a randomised, controlled, non-inferiority trial
}

\author{
A Metin Gülmezoglu, Pisake Lumbiganon, Sihem Landoulsi, Mariana Widmer, Hany Abdel-Aleem, Mario Festin, Guillermo Carroli, \\ Zahida Qureshi, João Paulo Souza, Eduardo Bergel, Gilda Piaggio, Shivaprasad S Goudar, John Yeh, Deborah Armbruster, Mandisa Singata, \\ Cristina Pelaez-Crisologo, Fernando Althabe, Peter Sekweyama, Justus Hofmeyr, Mary-Ellen Stanton, Richard Derman, Diana Elbourne
}

\section{Summary}

Background Active management of the third stage of labour reduces the risk of post-partum haemorrhage. We aimed to assess whether controlled cord traction can be omitted from active management of this stage without increasing the risk of severe haemorrhage.

Methods We did a multicentre, non-inferiority, randomised controlled trial in 16 hospitals and two primary healthcare centres in Argentina, Egypt, India, Kenya, the Philippines, South Africa, Thailand, and Uganda. Women expecting to deliver singleton babies vaginally (ie, not planned caesarean section) were randomly assigned (in a 1:1 ratio) with a centrally generated allocation sequence, stratified by country, to placental delivery with gravity and maternal effort (simplified package) or controlled cord traction applied immediately after uterine contraction and cord clamping (full package). After randomisation, allocation could not be concealed from investigators, participants, or assessors. Oxytocin $10 \mathrm{IU}$ was administered immediately after birth with cord clamping after 1-3 min. Uterine massage was done after placental delivery according to local policy. The primary (non-inferiority) outcome was blood loss of $1000 \mathrm{~mL}$ or more (severe haemorrhage). The non-inferiority margin for the risk ratio was $1 \cdot 3$. Analysis was by modified intention-to-treat, excluding women who had emergency caesarean sections. This trial is registered with the Australian and New Zealand Clinical Trials Registry, ACTRN 12608000434392.

Findings Between June 1, 2009, and Oct 30, 2010, 12227 women were randomly assigned to the simplified package group and 12163 to the full package group. After exclusion of women who had emergency caesarean sections, 11861 were in the simplified package group and 11820 were in the full package group. The primary outcome of blood loss of $1000 \mathrm{~mL}$ or more had a risk ratio of 1.09 (95\% CI 0.91-1.31) and the upper $95 \% \mathrm{CI}$ limit crossed the pre-stated non-inferiority margin. One case of uterine inversion occurred in the full package group. Other adverse events were haemorrhage-related.

Interpretation Although the hypothesis of non-inferiority was not met, omission of controlled cord traction has very little effect on the risk of severe haemorrhage. Scaling up of haemorrhage prevention programmes for non-hospital settings can safely focus on use of oxytocin.

Funding United States Agency for International Development and UN Development Programme/UN Population Fund/WHO/World Bank Special Programme of Research, Development and Research Training in Human Reproduction, Department of Reproductive Health and Research.

\section{Introduction}

Post-partum haemorrhage is a major cause of severe morbidity and maternal death, particularly in Africa and Asia, where nearly a third of pregnancy-related deaths are associated with haemorrhage. ${ }^{1}$ Most such deaths occur because of insufficient uterine contraction soon after birth. Two management packages for the third stage of labour are commonly used, known as active management and expectant management. ${ }^{2}$ In active management, several prophylactic interventions are applied in combination. WHO recommends administration of oxytocin soon after delivery of the baby, controlled cord traction, and delayed clamping and cutting of the cord until the health-care worker is ready to apply traction. ${ }^{3}$ Uterine massage after placental delivery is included in professional society guidelines. ${ }^{4}$ In expectant management, the interventions included in active management are withheld unless needed. Randomised trials ${ }^{5,6}$ of active versus expectant management have been done in hospital settings and they included early clamping and cutting of the cord in addition to the WHO components. Overall, the risk of post-partum haemorrhage was more than $60 \%$ lower with active management than with expectant management. The timing of cord clamping does not seem to play a significant part in blood loss.? Side-effects such as increased blood pressure, nausea, vomiting, and increased placental retention are generally attributed to the use of uterotonic ergot alkaloids.

WHO recommendations published in $2007^{3}$ advocated use of the full active management package, while
Lancet 2012; 379: 1721-27 Published Online March 6, 2012 DOI:10.1016/S01406736(12)60206-2

This publication has been corrected twice. The first corrected version first appeared at thelancet.com on May 4, 2012, and the second on July 20, 2012

See Comment page 1684

UNDP/UNFPA/WHO/World Bank Special Programme of Research, Development and Research Training in Human Reproduction, World Health Organization, Geneva, Switzerland

(A M Gülmezoglu MD, SLandoulsi MSc, M Widmer MSc, JP Souza MD, E Bergel PhD, G Piaggio PhD); Department of Obstetrics and Gynaecology, Faculty of Medicine, Khon Kaen University, Khon Kaen, Thailand (Prof P Lumbiganon MD); Department of Obstetrics and Gynaecology, Faculty of Medicine, Assiut University, Assiut, Egypt (Prof H Abdel-Aleem MD); Department of Obstetrics and Gynaecology, Philippine General Hospital, University of the Philippines, Manila, Philippines (M Festin MD, ( Pelaez-Crisologo MD); Centro Rosarino de Estudios Perinatales, Rosario, Argentina (G Carroli MD); Department of Obstetrics and Gynaecology, University of Nairobi, Nairobi, Kenya (Z Qureshi MD); Women's and Children's Health Research Unit, Jawaharlal Nehru Medical College, Belgaum, Karnataka, India (Prof S S Goudar MD); Harvard Medical School, Boston, MA, USA (Prof J Yeh MD); United States Agency for International Development, Washington, DC, USA (D Armbruster CNM, M-E Stanton MSN); Effective 
Care Research Unit, Eastern Cape Department of Health, Universities of Witwatersrand and Fort Hare, East London, South Africa (M Singata MBA, Prof J Hofmeyr MD); Institute for Clinical Effectiveness and Health Policy, Buenos Aires, Argentina (F Althabe MD); San Raphael of St Francis Hospital, Nsambya, Uganda (P Sekweyama MD); Department of Obstetrics and Gynaecology, Center for Women's and Children's Health Research, Christiana Care Health Services, Newark, DE, USA (R Derman MD); London School of Hygiene and Tropical Medicine, London, UK (Prof D Elbourne PhD)

Correspondence to: Dr A Metin Gülmezoglu, World Health Organisation, Department of Reproductive Health and Research,

Geneva CH-1211, Switzerland gulmezoglum@who.int acknowledging the absence of evidence for the effectiveness of some individual components. Estimation of the relative contribution of each of the components could help to determine the most effective way to use the intervention at different levels of health care or by healthcare workers with different skills. ${ }^{6}$ Some evidence suggests that the uterotonic component can be effective on its own. ${ }^{8,9}$ However, the contribution of controlled cord traction is largely unknown.

In controlled cord traction ${ }^{10}$ the birth attendant pushes the uterine fundus upwards with one hand while the other hand applies continuous traction on the umbilical cord to extract the placenta. ${ }^{11}$ Because the procedure requires manual skills, it has been recommended for use by skilled birth attendants only. However, if traction does not have a meaningful effect on blood loss then it could be omitted and a simplified package focusing mainly on the uterotonic could be recommended. Such a recommendation would have important implications for reducing resources required to train and deploy healthcare personnel attending childbirth and could expand access to effective care in places where staff shortages and physical barriers to access remain.

We aimed to find out whether a simplified approach to management of the third stage of labour without controlled cord traction is not less effective than the full active management package at reduction of severe blood loss (ie, $\geq 1000 \mathrm{~mL}$ ).

\section{Methods}

\section{Study design and patients}

We did a multicentre, non-inferiority, randomised controlled trial in 16 hospitals and two primary healthcare centres in Argentina, Egypt, India, Kenya, the Philippines, South Africa, Thailand, and Uganda. Before the trial started we assessed the existing policies at the study sites. With the exception of the two primary-care centres in India, all sites practised controlled cord traction. All sites used intramuscular or intravenous oxytocin $10 \mathrm{IU}$, although the timing of administration differed. In the Philippines, a policy of concurrent use of intramuscular oxytocin and ergometrine was in place before the trial. In Argentina and Kenya, cord clamping was described as delayed, whereas other sites were practising immediate cord clamping. Uterine massage after placental delivery was part of routine management in the Philippines and Thailand and reported as about $50 \%$ in Egypt. It was not part of routine policy in any of the other countries.

All women expecting to deliver vaginally at the participating hospitals were potentially eligible. They were excluded if they were first seen in advanced labour (>6 cm cervical dilatation), were not capable of giving consent because of an obstetric emergency, a mental disorder, or distress, or if they planned to have caesarean section, were minors without a guardian, or had twin pregnancies. The fetus had to be at a gestational age of viability according to local limits. Women were asked for written informed consent either before or early in labour. The trial protocol was approved by the WHO Ethics Review Committee and the local ethics committees of the participating institutions

\section{Randomisation and masking}

Random allocation took place as close to the anticipated vaginal delivery as possible. The random allocation sequence was computer generated centrally at WHO Randomisation was stratified by country and restricted with randomly varying blocks of six to eight. At each facility, a computer programmed with the random allocation sequence was provided and allocation was made once the woman's details were entered into the computer by local investigators. Each site had one spare computer in case of break-down or theft; if both failed the centre had to revert to sealed opaque envelopes as the back-up option. Centres in Egypt and Kenya had to revert to sealed opaque envelopes because of break-down and theft midway through the trial. Neither the investigators nor participants could be masked to the interventions or outcomes.

\section{Procedures}

In both groups of the trial, intramuscular oxytocin 10 IU was administered as soon as possible after birth, preferably within $1 \mathrm{~min}$. If a woman had an intravenous line, oxytocin diluted in $100 \mathrm{~mL}$ saline could be administered through the line for $5 \mathrm{~min}$. The cord was clamped and cut after manual or visual observation of a uterine contraction, usually around 1-3 min after delivery of the baby. Collection of lost blood was initiated immediately after birth of the baby by passing a drape under the woman's buttocks. ${ }^{12}$ Blood collected in the drape was put in a bucket, weighed on a digital scale together with the drape, and the amount recorded in grams. Trial interventions were administered by the staff who managed the deliveries. For the analysis, the weight of the drape was subtracted and the weights were converted to volumes; the value in grams was divided by 1.06 (blood density in $\mathrm{g} / \mathrm{mL}$ ). ${ }^{13}$

The experimental intervention assessed in the trial was the simplified package, in which placental delivery was allowed to occur with the aid of gravity and maternal effort. The birth attendant was expected to note the signs of placental separation such as a gush of blood, uterine contraction, lengthening of the umbilical cord, and visualisation of the placenta in the vagina, and then encourage the woman to cough or push. The control was the full package, in which controlled cord traction was applied immediately after observation of a uterine contraction. The cord was clamped and cut as described for the simplified package. The full package practised in the trial was applied as in other active management trials except for timing of cord clamping. However, available evidence suggests that the timing of cord clamping is not likely to have a major effect on blood loss.? The protocol required that after placental delivery the uterine 
fundus was rubbed and any clots expressed. Because policies for uterine massage varied and no evidence to support or refute its role is available, individual sites were allowed to implement it or not according to their existing routine. The protocol stated that, if the procedure was used, the uterus should be massaged gently until it contracted and then every $15 \mathrm{~min}$ for up to $2 \mathrm{~h}$. Adherence to allocated treatment was defined as hands-off management of the cord in the simplified package group and application of cord traction within $30 \mathrm{~min}$ of delivery in the full package group.

The primary (non-inferiority) outcome was severe postpartum haemorrhage defined as measured blood loss of $1000 \mathrm{~mL}$ or more at $1 \mathrm{~h}$ and up to $2 \mathrm{~h}$ for women who continued to bleed after $1 \mathrm{~h}$. The secondary (superiority) outcomes were blood transfusion, use of additional uterotonics, blood loss of $500 \mathrm{~mL}$ or more, maternal death, manual placental removal, surgical procedures (hysterectomy, ligation of vessels), the composite outcome of maternal death or severe morbidity (admission to intensivecare unit, hysterectomy, blood loss of $2000 \mathrm{~mL}$ or more, uterine inversion), and initiation of breastfeeding.

The Data Safety and Monitoring Committee met three times during the course of the project. The Committee reviewed two interim analyses according to the HaybittlePeto rule $\mathrm{e}^{14}$ with a superiority criterion on the primary outcome. The committee advised the trial Steering Committee to continue recruitment on both occasions.

\section{Statistical analysis}

We aimed to assess non-inferiority of the simplified package compared with the full package in terms of efficacy within a pre-stated non-inferiority margin. We chose the non-inferiority margin by examining the effect of the gold standard, in this case full active management, compared with expectant management (control) in previous trials., ${ }^{515}$ Estimates of severe post-partum haemorrhage with active and expectant management were taken from published and unpublished data of WHO studies in which post-partum blood loss was measured. ${ }^{5,9,16}$ On the basis of those data, we assumed a $1.5 \%$ risk of severe haemorrhage with the full package. The severe haemorrhage rate with expectant management was based on data available from earlier trials. ${ }^{5,15}$ Values between $3.0 \%$ and $4.0 \%$ were judged realistic. For sample size estimates, we therefore assumed a $3 \%$ risk of severe haemorrhage with expectant management. The simplified package was assumed to preserve $70 \%$ of the benefit of the full active management package. With these assumptions, a trial of 22908 women would have $80 \%$ power to show non-inferiority of the simplified package within $0.45 \%$ of the severe haemorrhage rate for the full package (ie, $[1 \cdot 0-0 \cdot 7] \times[3 \cdot 0-1 \cdot 5]$, with a two-sided $95 \% \mathrm{CI}$, and an $\alpha$ of $2.5 \%$ ). In relative terms, this gives a margin of non-inferiority of 1.3 (ie $[1 \cdot 5+0 \cdot 45] / 1 \cdot 5=1 \cdot 95 / 1 \cdot 5)$. The non-inferiority hypothesis was used for the primary outcome. For the secondary endpoints superiority hypotheses were used. SAS version 9.1 was used for all analyses.

We identified two analysis populations: intention-totreat population-all enrolled women assessed within groups as randomised, irrespective of adherence-and modified intention-to-treat population-as intention-totreat population, but excluding women having a caesarean section after randomisation. Our primary analysis and interpretation are based on the modified intention-totreat population. We chose to focus on this population because for women having a caesarean section (whether elective or emergency), the trial interventions could not be implemented, nor the primary outcome assessed, in the same way as with a vaginal delivery. Elective caesarean section was an exclusion criterion but some emergency caesarean sections occurred after randomisation.

Although some researchers have suggested that in non-inferiority trials per-protocol analysis provides a more conservative interpretation, ${ }^{17}$ this notion has been disputed..$^{18}$ In this trial, women with much blood loss in the simplified group were more likely to receive cord traction and other interventions to remove the placenta than were women with little blood loss. Because women with great blood loss would be excluded in a per-protocol analysis, the results would favour the simplified package group, and push the results towards erroneous non-inferiority.

Because the effects of uterine massage with or without the full package were unknown, we planned stratified analysis according to the policy of use or non-use of uterine massage. Finally, we did sensitivity analyses with and without the data set from the Philippines. We

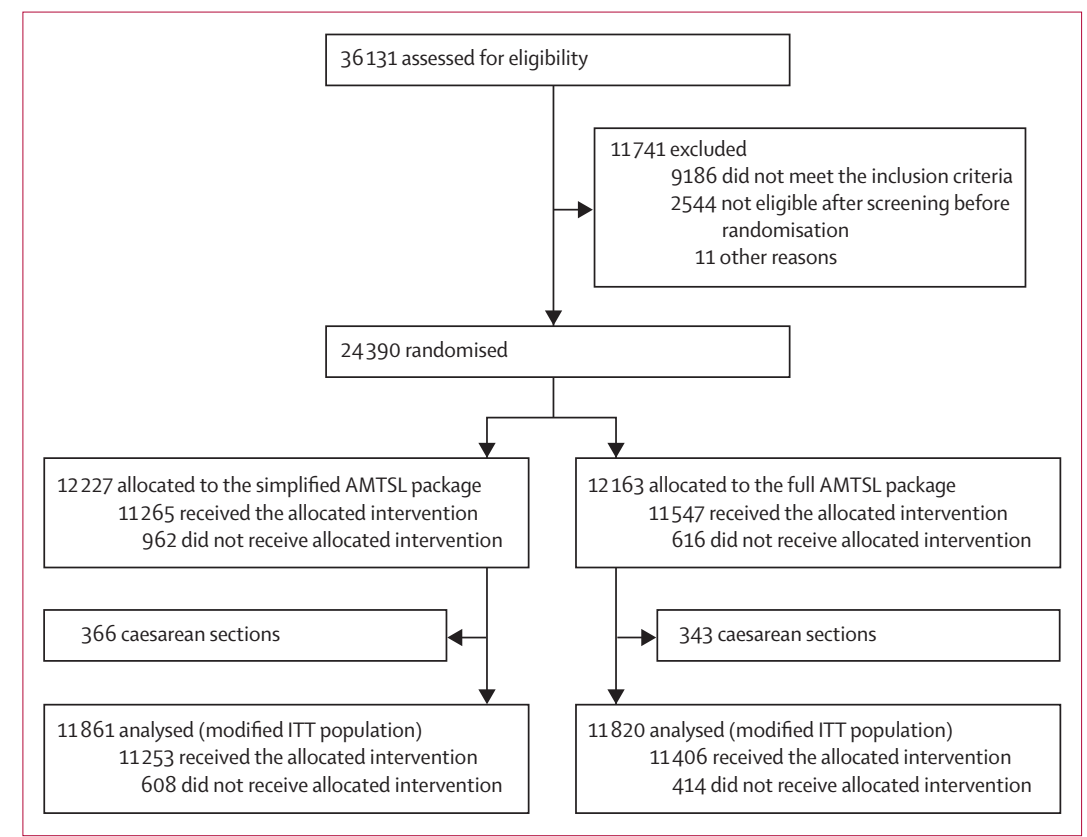

Figure: Trial profile

AMTSL=active management of the third stage of labour. ITT=intention to treat. 


\begin{tabular}{|lcc|}
\hline & Simplified package & Full package \\
\hline Age $($ years) & \\
\hline Primigravidae & $25 \cdot 3(5 \cdot 6)$ & $25 \cdot 3(5 \cdot 6)$ \\
\hline Duration of gestation $\geq 37$ weeks & $5362 / 11823(45 \%)$ & $5476 / 11799(46 \%)$ \\
\hline Labour induced or augmented & $10713 / 11815(91 \%)$ & $10620 / 11797(90 \%)$ \\
Spontaneous cephalic vaginal delivery & $5923 / 11815(50 \%)$ & $5796 / 11795(49 \%)$ \\
\hline Perineal trauma (episiotomy or tear requiring suture) & $11266 / 11818(95 \%)$ & $11240 / 11795(95 \%)$ \\
Birthweight $(\mathrm{g}) \dagger$ & $7667 / 11816(65 \%)$ & $7616 / 11791(65 \%)$ \\
\hline Baby alive & $3069(538)$ & $3054(544)$ \\
\hline
\end{tabular}

Data are $\mathrm{n} / \mathrm{N}$ (\%) or mean (SD). Denominators vary because of missing data. ${ }^{*} \mathrm{~N}=11861$ for the simplified group, and 11820 for the full package group. $\uparrow \mathrm{N}=11818$ for the simplified group, and 11797 for the full package group.

Table 1: Characteristics of women at trial entry and delivery for the modified intention-to-treat population

\begin{tabular}{|c|c|c|}
\hline & Simplified package & Full package \\
\hline \multicolumn{3}{|l|}{ Adherence } \\
\hline Cord management according to protocol ${ }^{*} \dagger$ & $11253(95 \%)$ & $11406(97 \%)$ \\
\hline Oxytocin administered $\dagger$ & $11779(99 \%)$ & $11751(99 \%)$ \\
\hline Delayed cord clamping and cutting $\dagger$ & 9321 (79\%) & $9242(78 \%)$ \\
\hline \multicolumn{3}{|c|}{ Interventions used for final delivery of the placentał } \\
\hline Hands off (maternal effort) $§$ & $10668(91 \%)$ & $833(7 \%)$ \\
\hline Controlled cord traction $\boldsymbol{\Phi}$ & $731(6 \%)$ & $11389(97 \%)$ \\
\hline Umbilical vein injection $\|$ & $10(<1 \%)$ & $10(<1 \%)$ \\
\hline Manual removal || & $153(1 \%)$ & $105(<1 \%)$ \\
\hline Curettage $\|$ & $165(1 \%)$ & $98(<1 \%)$ \\
\hline Hysterectomy\|l & $2(<1 \%)$ & $6(<1 \%)$ \\
\hline Left in situ because of severe adherence** & $2(<1 \%)$ & $6(<1 \%)$ \\
\hline Placenta and baby delivered simultaneously ${ }^{\dagger} \dagger$ & $29(<1 \%)$ & $23(<1 \%)$ \\
\hline \multicolumn{3}{|c|}{$\begin{array}{l}\text { Data are } \mathrm{n}(\%) \text {. Denominators vary because of missing data. *Hands-off in the simplified package and controlled cord } \\
\text { traction in the first } 30 \text { min in the full package. }+\mathrm{N}=11861 \text { for the simplified package, and } 11820 \text { for the full package. } \\
\ddagger \text { More than one intervention could be used for any one patient. } \$ \mathrm{~N} \text { for hands off is } 11776 \text { for the simplified package, } \\
\text { and } 11763 \text { for the full package. } \uparrow \mathrm{N} \text { for controlled cord traction is } 11777 \text { for the simplified package, and } 11766 \text { for the } \\
\text { full package. IIN for umbilical vein injection, manual removal, curettage, and hysterectomy is } 11814 \text { for the simplified } \\
\text { package, and } 11794 \text { for the full package. }{ }^{*} \mathrm{~N} \text { for left in situ is } 11813 \text { for the simplified package and } 11792 \text { for the full } \\
\text { package. }+\dagger \mathrm{N} \text { for delivered simultaneously is } 11814 \text { for the simplified package, and } 11797 \text { for the full package. }\end{array}$} \\
\hline
\end{tabular}

decided on the sensitivity analysis after the protocol was written but before the analysis. In the Philippines, both study sites were large, high-volume hospitals and frequent closures occurred because of repairs and infections in the neonatal units that led to interruptions in recruitment and loss of study forms (140 of 2527 [5 - 5\%] in the simplified package group and 137 of 2524 [5.4\%] in the full package group). When examining the quality of data from the Philippines, we noted that in one of the two sites, the routine policy of oxytocin and ergometrine administration from before initiation of the study continued during the trial. For these reasons, and especially in view of the association of ergometrine with placental retention identified in published work, ${ }^{5}$ the trial steering committee decided that the sensitivity analyses were justified. This trial is registered with the Australian and New Zealand Clinical Trials Registry, ACTRN 12608000434392.

\section{Role of the funding source}

The study was supported by the United States Agency for International Development and UN Development Programme/UN Population Fund/WHO/World Bank Special Programme of Research, Development and Research Training in Human Reproduction, Department of Reproductive Health and Research, WHO. The sponsors had no role in data collection, analysis, or interpretation of the data, the writing of the report, or the decision to submit for publication. All authors had access to the analysis plan, the outputs of that analysis and could see the data if they wished to do so. All authors participated in the final discussion and approved the submission.

\section{Results}

The figure shows the trial profile. Enrolment took place between June 1, 2009, and Oct 30, 2010. 3455 women were in Argentina, 3527 in Egypt, 2165 in India, 4005 in Kenya, 5051 in the Philippines, 1277 in South Africa, 3181 in Thailand, and 1729 in Uganda. Table 1 shows patient characteristics at trial entry and delivery. Adherence to the oxytocin and controlled cord traction components was high $(>90 \%)$, with overall package adherence reduced mainly because of the timing of cord clamping and cutting (table 2). Rates of early cord clamping were particularly high in some of the Thai facilities because of hospital policies. Most sites did not practise uterine massage regularly, except Egypt (2507 of 3518 cases, $99 \cdot 7 \%)$, Thailand ( 2790 of $2976,93 \cdot 7 \%)$, and Uganda (1715 of 1723 cases, 99.8\%). In all other sites uterine massage was used in less than $10 \%$ of cases. Some differences between policies before and at the end of the trial are to be expected because the principal investigators attempted to bring their policies in line with trial procedures. About $6 \%$ of all women allocated to the simplified package eventually needed controlled cord traction (table 2). We were not able to differentiate whether their placentas were truly retained or whether the total included women whose placentas were already separated but not expelled by maternal efforts.

Table 3 shows primary and secondary outcomes. For the primary endpoint, the upper range of the $95 \% \mathrm{CI}$ lies slightly above the non-inferiority limit $(1 \cdot 30)$. The risk of the secondary outcome of post-partum haemorrhage was higher in women given the simplified package than in those given the full package (risk ratio 1.07, 95\% CI $1 \cdot 00-1 \cdot 14$, superiority hypothesis). The rate of manual placental removal was significantly higher in the simplified package group (table 3). Mean blood loss was about $11 \mathrm{~mL}$ greater and the third stage about $7 \mathrm{~min}$ longer with the simplified package than with the full package. One case of uterine inversion occurred in the full package group. Other adverse events were haemorrhage-related.

In the sensitivity analysis excluding the Philippines (table 4), the summary estimates had slightly larger CIs, and the statistically significant difference in the rate of 


\begin{tabular}{|c|c|c|c|c|}
\hline & Simplified package & Full package & $\%$ risk difference $(95 \% \mathrm{Cl})$ & Risk ratio $(95 \% \mathrm{Cl})$ \\
\hline Blood loss $\geq 1000 \mathrm{~mL}^{*}$ & $239(2 \%)$ & $219(2 \%)$ & $0.17(-0.19$ to 0.53$)$ & 1.09 (0.91 to 1.31$)$ \\
\hline Blood loss $\geq 500 \mathrm{~mL}^{*}$ & $1598(14 \%)$ & $1493(13 \%)$ & $0.90(0.03$ to 1.78$)$ & $1.07(1.00$ to 1.14$)$ \\
\hline Blood loss in $\mathrm{mL} \dagger$ & 282 & 271 & $10 \cdot 8(4.7$ to $16 \cdot 9)$ & .. \\
\hline Additional uterotonics $¥$ & $2434(21 \%)$ & $2390(20 \%)$ & $0.34(-0.69$ to 1.37$)$ & $1.02(0.97$ to 1.07$)$ \\
\hline Blood transfusion § & $62(<1 \%)$ & $55(<1 \%)$ & $0.06(-0.12$ to 0.24$)$ & $1.12(0.78$ to 1.62$)$ \\
\hline Manual removal of placenta & $153(1 \%)$ & $105(<1 \%)$ & $0.40(0.14$ to 0.67$)$ & 1.45 (1.14 to 1.86$)$ \\
\hline Third stage duration in $\mathrm{min} \|$ & 12.6 & $6 \cdot 1$ & $6.5(6.2$ to 6.8$)$ & .. \\
\hline Maternal death** & $2(<1 \%)$ & $1(<1 \%)$ & $0.01(-0.02$ to 0.04$)$ & $2.00(0.18$ to 22.0$)$ \\
\hline Additional surgical procedures $+\dagger$ & $2(<1 \%)$ & $9(<1 \%)$ & $-0.06(-0.11$ to 0.00$)$ & $0.22(0.05$ to 1.03$)$ \\
\hline Maternal death or severe morbidity $\neq \neq$ & $20(<1 \%)$ & $31(<1 \%)$ & $-0.09(-0.22$ to 0.03$)$ & 0.65 (0.37 to 1.13$)$ \\
\hline Baby put to breast within $30 \min \$ \Omega$ & $10565(90 \%)$ & $10532(90 \%)$ & 0.14 (-0.62 to 0.91$)$ & 1.00 (0.99 to 1.01$)$ \\
\hline \multicolumn{5}{|c|}{ 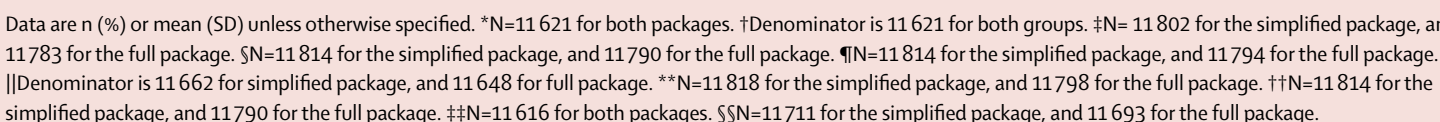 } \\
\hline
\end{tabular}

\begin{tabular}{|c|c|c|c|c|}
\hline & Simplified package & Full package & $\%$ risk difference $(95 \% \mathrm{Cl})$ & Risk ratio $(95 \% \mathrm{Cl})$ \\
\hline Blood loss $\geq 1000 \mathrm{~mL}^{*}$ & $153(2 \%)$ & $140(2 \%)$ & $0.14(-0.21$ to 0.49$)$ & 1.09 (0.87 to 1.37$)$ \\
\hline Blood loss $\geq 500 \mathrm{~mL}^{*}$ & $987(11 \%)$ & $927(10 \%)$ & $0.65(-0.22$ to 1.51$)$ & 1.07 (0.98 to 1.16$)$ \\
\hline Blood loss $(\mathrm{mL}) \dagger$ & 266 & 256 & $10 \cdot 2(3.9$ to $16 \cdot 4)$ & .. \\
\hline Additional uterotonics $\ddagger$ & $1026(11 \%)$ & $962(10 \%)$ & $0.66(-0.21$ to 1.54$)$ & $1.07(0.98$ to 1.16$)$ \\
\hline Blood transfusion $\S$ & $50(<1 \%)$ & $34(<1 \%)$ & $0.17(-0.02$ to 0.36$)$ & $1.47(0.95$ to 2.27$)$ \\
\hline Manual removal of placenta & $62(<1 \%)$ & $64(<1 \%)$ & $-0.02(-0.25$ to 0.21$)$ & $0.97(0.68$ to 1.37$)$ \\
\hline Third stage duration $(\mathrm{min}) \|$ & $11 \cdot 4$ & $6 \cdot 2$ & $5.2(4.9$ to 5.5$)$ & .. \\
\hline Maternal death ${ }^{* *}$ & $1(<1 \%)$ & $1(<1 \%)$ & $0.00(-0.03$ to 0.03$)$ & $1.00(0.06$ to 16.0$)$ \\
\hline Additional surgical procedures $\$$ & 0 & $3(<1 \%)$ & $-0.03(-0.07$ to 0.00$)$ & .. \\
\hline Maternal death or severe morbidity ${ }^{\dagger}$ & $12(<1 \%)$ & $15(<1 \%)$ & $-0.03(-0.14$ to 0.08$)$ & 0.80 (0.37 to 1.71$)$ \\
\hline Baby put to breast within $30 \mathrm{~min} \ddagger \ddagger$ & $8571(91 \%)$ & $8566(91 \%)$ & $-0.01(-0.85$ to 0.82$)$ & 1.00 (0.99 to 1.01$)$ \\
\hline \multicolumn{5}{|c|}{ 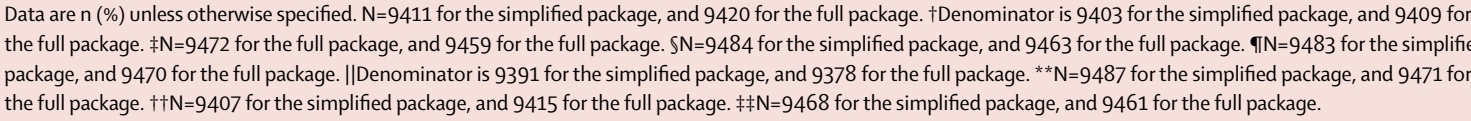 } \\
\hline
\end{tabular}

manual placental removal was no longer apparent. Mean blood loss was still greater and the third stage longer with the simplified package than with the full package. We recorded uterine massage after placental delivery and did a pre-specified subgroup analysis of the primary and secondary outcomes with or without uterine massage (data not shown). The interaction test did not suggest that the outcomes differed according to this policy $(\mathrm{p}=0 \cdot 61)$.

\section{Discussion}

Our findings suggest that omission of cord traction results in very little, if any, increased risk of severe haemorrhage. The primary outcome of blood loss of $1000 \mathrm{~mL}$ or more has a risk ratio of 1.09 (95\% CI 0.91 to 1.31 ) and the upper $95 \% \mathrm{CI}$ limit crosses the pre-stated non-inferiority margin of 1.30 (appendix). If the upper limit had been below 1.30 we would have declared the simplified package non-inferior to the full package. ${ }^{19}$ The estimated number needed to harm (the reciprocal of the risk difference $0 \cdot 17 \%$ ) is 581 (one-sided $97.5 \%$ CI 189 to $\infty$ ), indicating that for every 581 women receiving the simplified package, one additional woman would have a severe haemorrhage than if all received the full package. We draw two inferences from these results: first, controlled cord traction is safe and its use can be continued in settings in which it is routinely practised; and second, the main component of active management is the uterotonic and in settings in which the full package cannot be used safely, focus should be on the uterotonic component (panel).

The higher rate of manual removal with the simplified package is probably attributable to the high use of ergometrine as prophylaxis in one of the Philippines See Online for appendix 
Panel: Research in context

\section{Systematic review}

We searched PubMed and the Cochrane Library with the terms "postpartum hemorrhage" and "randomised controlled trial" for trials and systematic reviews assessing controlled cord traction. We indentified one review ${ }^{20}$ at protocol stage and one pilot randomised trial, ${ }^{13}$ which included 200 women and suggested a potential protective effect of controlled cord traction on blood loss. The date of the last search was Feb 7, 2012.

\section{Interpretation}

Our study provides the largest evidence so far of the effect of controlled cord traction as part of active management.

Controlled cord traction adds only marginally to the beneficial effect of the full package. Because the main component of the management package is oxytocin, in settings in which no skilled birth attendants are present to give the full package, efforts should focus on the uterotonic (primarily oxytocin) to reduce post-partum haemorrhage.

sites as suggested by the sensitivity analysis and earlier systematic review. ${ }^{5}$

Our findings challenge the concept of active management of the third stage of labour as a package of interventions. Effective prevention of post-partum haemorrhage can be accomplished by an intramuscular injection of oxytocin $10 \mathrm{IU}$ after delivery of the baby and present evidence suggests that the uterotonic component of the active management package is the essential component.

The strengths of our study were its size and the diversity of settings of the study sites. We used a secure method of allocation concealment that allocated the women only after specific data were entered. Furthermore, the large number of participants allowed us to implement the study at a realistic scale in line with the prevalence of severe postpartum haemorrhage recorded in other work. The data for measured blood loss were collected consistently across the trial sites. The adherence to trial intervention in terms of cord management was high-in previous trials of active versus expectant management, adherence with cord management was between $46 \%$ and $99 \%$. $^{5}$ Because of the nature of the intervention we could not do the trial in a double-blind manner. We acknowledged the potential for bias and attempted to minimise this risk by providing standard training for staff who were going to implement the trial interventions. However, we could not make blind outcome assessments.

Our study is relevant both to health-facility settings and to those in which health-care personnel do not have the skills to apply controlled cord traction safely. However, we could not have done the trial in a non-facility setting precisely because such personnel are not available. The issue investigated is also relevant to settings in which skilled attendants are available but women prefer a hands-off mode of placental delivery. Our findings suggest such placental delivery can be safely offered in those circumstances.

Recruitment interruptions and the high number of deliveries in the Philippines resulted in loss of about 5\% of forms. However, those losses were similar in both groups of the trial. The high rates of ergometrine use in one of the Philippine hospitals was another justification for the sensitivity analysis, which supported the hypothesis that the overall increase in manual removal could be attributable to ergometrine use. However, this analysis was post hoc and the increase in manual removal could be attributable to other unknown factors.

Our findings have several implications for clinical practice. Simplification of the active management of third stage of labour is possible: an intramuscular injection of oxytocin 10 IU after delivery of the baby should be regarded as the primary intervention for prevention of haemorrhage. Injections are increasingly being used in settings in which skilled birth attendants are not available but a trained health worker is present. In such settings, oxytocin should be used as the routine uterotonic for prevention of post-partum haemorrhage even if controlled cord traction cannot be implemented. Such a policy could also result in cost savings by eliminating the need for training in cord traction skills. In settings in which skilled birth attendants are available, the full package of oxytocin and controlled cord traction should be preferred especially if the shortest possible third-stage duration is desirable. Because a few women will eventually require controlled cord traction with the simplified package and it is the first procedure to be attempted in case of retained placenta, we believe that teaching of controlled cord traction in medical and midwifery curricula should continue.

Our study likewise has implications for future research. A substantial proportion of maternal deaths from haemorrhage take place in settings in which skilled birth attendants and facility-based care are not available. Our findings strengthen the need to focus on strategies to scale up the use of oxytocin in peripheral health-care settings as the primary component of active management of the third stage of labour. In this context, the use of task-shifting strategies to expand access should be considered and assessed. Such scaling-up efforts could be aided by use of prophylactic oxytocin in a prefilled, compact, auto-disabled device system. A clusterrandomised trial to investigate the effects of this system on post-partum haemorrhage at community level in Ghana is under way (NCT01108289). In settings in which no injection is possible, the role of misoprostol should be assessed with similarly rigorous research.

We were not able to examine any possible role of uterine massage in reducing blood loss in this trial. Uterine massage remains one component of third-stage management that can be implemented without any need for technology or skilled birth attendants. However, it has 
not been rigorously assessed so far. ${ }^{21}$ The variation in use of uterine massage across study sites suggests that uncertainty remains among practitioners and a thorough investigation of the preventive and therapeutic use of this intervention would be timely.

\section{Contributors}

The idea for the study and its design were discussed and agreed at an international meeting in 2007. AMG led the writing of the draft protocol, which was commented on and finalised by the principal investigators and other members of the Steering Committee. AMG coordinated the study with support from staff in WHO (GP, MW, JPS, SL, and EB), drafted the analysis plan with GP and DE, wrote the draft report and together with other members of the WHO team incorporated comments and suggestions from the Steering Committee members. SL did data monitoring in WHO, Switzerland, and GP analysed the data with support from SL. Site principal investigators and co-principal investigators commented on the protocol; coordinated the study in their settings; attended Steering Committee meetings; read, commented, and approved the final analysis and the article. DA, M-ES, JY, and RD were members of the Steering Committee and contributed to the protocol, participated at the SC meetings, and contributed to the final report. FA attended Steering Committee meetings, coordinated the data monitoring team in Buenos Aires, Argentina, and contributed to the protocol and the final article. DE is the Chair of the Steering Committee, contributed to the protocol, chaired Steering Committee meetings, commented on the analysis plan, drafted the final report, and interacted with the Data Safety and Monitoring Committee.

\section{Investigators}

Trial Coordinating Unit: A M Gülmezoglu, S Landoulsi, M Widmer, G Piaggio, J P Souza, E Bergel, A-L Fersurella, A Seuc, C C Kiener. Data monitoring team: L Gibbons, L Gutiérrez, F Althabe. Steering Committee-UK: D Elbourne (Chair); Argentina: G Carroli, F Althabe; Egypt: H Abdel-Aleem; India: S S Goudar, Kenya: Z Qureshi; Philippines: C Pelaez-Crisologo, M Festin; South Africa: G J Hofmeyr, M Singata; Thailand: P Lumbiganon; U Peter Sekweyama; USA: D Armbruster, M-E Stanton, J Yeh, R Derman.

Data Safety and Monitoring Committee-South Africa: R Pattinson (Chair); Ghana: J Gyapong; UK: R McCandlish; USA: K Schulz.

\section{Country teams}

Argentina G Carroli (Principal Investigator), M Paccioco, F Farandato, M Toribio, A L Beccaria, G Mandrilli (Hospital Eva Peron); S Carbognani, N Cabrejas, C A Nardin, P Grantile, M Marini (Hospital Maternidad Martin); M Raffagnini, F Boeykens, M Ruiz, J Ondategui, I Lopez (Hospital Roque Saenz Peña); L Campodonico, F Candio, Y Sguassero, P Mazzei, F Burgueño, H Gamerro, L Minoldo, D Giordano (CREP). Egypt $\mathrm{H}$ Abdel-Aleem (Principal Investigator), A Ibraheem, M A-A Ahmad, M Kahleefa. India S Goudar (Principal Investigator), B S Kodkany (Co-Principal Investigator), Y V Pujar, H A Dhumale. Kenya Z Qureshi (Principal Investigator), C S Kigondu, G Jaldesa, K Kamau, A Adhiambo, S Guyo, M Okul, L Ngatia, M Waweru, F Mbage, and staff of labour ward Kenyatta National Hospital. Philippines C Pelaez-Crisologo (Principal Investigator), M Festin, A Layug, M Ong, H Juban, E Torralba, J Abaleta, J Carasco, H Bellin, D Grace Luz, J Lumpay. South Africa G J Hofmeyr (Principal Investigator), S Mngcozelo, A Aku, X Williams, K C Seloka, N Mshweshwe. Thailand P Lumbiganon (Principal Investigator), M Laopaiboon, J Thinkhamrop, J Wachirapakorn, C Supanavong (Khon Kaen University); U Swadpanich, T Siriwachirachai, O Rujirakul, B Sanreang (Khon Kaen Hospital); B Suwannachat, B Chaikumming, S Ritsuwan (Kalasin Hospital); N Winiyakul, K Vivatpatanakul (Health Promotion Centre Region 6). Uganda P Sekweyama (Principal Investigator), P Okong.

\section{Conflicts of interest}

We declare that we have no conflicts of interest.

\section{Acknowledgments}

We thank the women who took part in the trial and the doctors, nurses, and midwives in the participating hospitals for making the project possible. We also thank Matthews Mathai and Kathy Herschderfer for participating in trial meetings and Neal Brandes and Lily Kak for support throughout the project and comments on the report. We thank Novartis for donating the oxytocin ampoules used in the trial. This trial was supported by the United States Agency for International Development (USAID) and UNDP/UNFPA/WHO/World Bank Special Programme of Research, Development and Research Training in Human Reproduction of WHO. Some of the authors are staff of the sponsor organisations. The interpretation and conclusions are those of the individual authors and not necessarily those of the sponsors.

\section{References}

1 Khan KS, Wojdyla D, Say L, Gülmezoglu AM, Van Look PFA. WHO analysis of causes of maternal death: a systematic review. Lancet 2006; 367: 1066-74.

2 Festin MR, Lumbiganon P, Tolosa JE, et al. International survey on variations in practice of the management of the third stage of labour. Bull World Health Organ 2003; 81: 286-91.

3 WHO. WHO Recommendations for the prevention of postpartum haemorrhage. Geneva: World Health Organization, 2007.

4 Clinical Practice Obstetrics Committee. Active management of the third stage of labour: prevention and treatment of postpartum hemorrhage: No. 235 October 2009 (Replaces No. 88, April 2000). Int J Gynecol Obstet 2010; 108: 258-67.

5 Begley CM, Gyte GML, Murphy DJ, Devane D, McDonald SJ, McGuire W. Active versus expectant management for women in the third stage of labour. Cochrane Database of Syst Rev 2010; 7: CD007412.

6 Keirse MJC. What does prevent postpartum haemorrhage? Lancet 1998; 351: 690-92.

7 McDonald SJ, Middleton P. Effect of timing of umbilical cord clamping of term infants on maternal and neonatal outcomes. Cochrane Database Syst Rev 2008; 2: CD004074.

8 Cotter A, Ness A, Tolosa JE. Prophylactic oxytocin for the third stage of labour. Cochrane Database Syst Rev 2001; 4: CD001808.

9 Gulmezoglu AM, Forna F, Villar J, Hofmeyr GJ. Prostaglandins for preventing postpartum haemorrhage. Cochrane Database Syst Rev 2007; 3: CD000494.

10 Spencer PM. Controlled cord traction in management of the third stage of labour. BMJ 1962; 1: 1728-32.

11 WHO. Managing complications in pregnancy and childbirth. World Health Organization, 2007. http://www.who.int/reproductivehealth/ publications/maternal_perinatal_health/9241545879/en/index.html (accessed Feb 19, 2011).

12 Patel A, Goudar SS, Geller SE, et al. Drape estimation vs. visual assessment for estimating postpartum hemorrhage. Int J Gynaecol Obstet 2006; 93: 220-24.

13 Althabe F, Aleman A, Tomasso G, et al. A pilot randomized controlled trial of controlled cord traction to reduce postpartum blood loss. Int J Gynaecol Obstet 2009; 107: 4-7.

14 Piantadosi S. Clinical trials: a methodological perspective. 2nd edn. Hoboken, NJ: John Wiley \& Sons, 1997.

15 Gülmezoglu AM, Widmer M, Merialdi M, et al. Active management of the third stage of labour without controlled cord traction: a randomized non-inferiority controlled trial. Reprod Health 2009; 6: 2

16 Widmer M, Blum J, Hofmeyr GJ, et al. Misoprostol as an adjunct to standard uterotonics for treatment of post-partum haemorrhage: a multicentre, double-blind randomised trial. Lancet 2010; 375: 1808-13.

17 ICH Steering Committee. Statistical Principles for Clinical Trials (E9). International Conference on Harmonisation of Technical Requirements for Registration of Pharmaceuticals for Human Use. International Conference on Harmonisation 1998. http://www.ich. org/products/guidelines/efficacy/article/efficacy-guidelines.html (accessed Feb 19, 2011).

18 Garrett A. Therapeutic equivalence: fallacies and falsification Stat Med 2003; 22: 741-62.

19 Piaggio G, Elbourne DR, Altman DG, Pocock SJ, Evans SJ. Reporting of noninferiority and equivalence randomized trials: an extension of the CONSORT statement. JAMA 2006; 295: 1152-60.

20 Mshweshwe NT, Hofmeyr GJ, Gülmezoglu AM. Controlled cord traction for the third stage of labour. Cochrane Database Syst Rev 2009; 4: CD008020.

21 Hofmeyr GJ, Abdel-Aleem H, Abdel-Aleem MA. Uterine massage for preventing postpartum haemorrhage. Cochrane Database Syst Rev 2008; 3: CD006431. 